\title{
Pilotage de réseaux stratégiques dans les marchés publics
}

\author{
Par Olivier Mamavi ${ }^{a, b}$ \\ ${ }^{a} / C D / L A R A$ \\ 'I.A.E. de l'Université de Poitiers / CEREGE
}

\section{Résumé}

Cet article montre comment une entreprise peut identifier les réseaux qui lui permettront de former des coalitions pour obtenir des marchés publics. Pour cela, nous avons représenté, à travers un graphe, le système des relations d'alliances de l'année 2008. Nous avons détecté, dans ce graphe, 1360 réseaux stratégiques dont l'organisation révèle, d'une part, des caractéristiques identiques à des réseaux sociaux, et, d'autre part, le rôle d'acteurs dominants dans l'accès aux ressources. À partir de ces résultats, nous proposons une cartographie des réseaux qui permet d'envisager de nouvelles applications d'intelligence économique.

(c) 2012 Lavoisier SAS. All rights reserved

Mots clés : réseaux stratégiques, intelligence économique, marché public, cartographie, théorie des réseaux sociaux.

\section{Abstract}

Management of strategic networks in public procurement. This study shows the process used by a company to find out business network in order to create collaboration to obtain public contract. We represented, with a graph, the alliance system of 2008 year. We identified first, 1360 strategic networks which structural organization looks like social network and second the main resources providers. These results prompt us to propose a network map which allow the identification of new application of competitive intelligence.

(c) 2012 Lavoisier SAS. All rights reserved

Keywords: strategic networks, business intelligence, public contractor, mapping, social networks theory. 


\section{Introduction}

En France, la commande publique de l'État, des collectivités territoriales et des organismes publics représente plus de 80 milliards d'euros par an ${ }^{1}$. Près de $8 \%$ de ces transactions sont obtenues dans le cadre de co-traitance. La co-traitance est la situation dans laquelle des entreprises qui répondent à un appel d'offres peuvent s'allier à travers un groupement d'entreprises, pour proposer une réponse collective lorsqu'elles ne possèdent pas, seules, les compétences et les ressources suffisantes.

Obtenir des marchés publics en co-traitance revient à former des coalitions d'entreprises. Une coalition est une alliance stratégique (Jolly, 2001), entre plusieurs entreprises indépendantes, qui est temporaire. En fait, la coalition est un accord de partenariat, sans structure juridique, ni apports capitalistiques, dont le but consiste à organiser une coopération, non hiérarchique, pour partager ou diffuser des ressources, afin de répondre aux besoins d'un projet (Lemieux, 1998).

Mais l'organisation d'une coalition n'est pas naturelle entre des entreprises aux intérêts parfois divergents. Alors, comment une entreprise peut-elle analyser son environnement pour former une coalition et obtenir des marchés publics?

Dans la littérature en sciences de gestion, de nombreux auteurs se sont intéressés aux coalitions d'entreprises et plus généralement à la formation des alliances (Kogut, 1988); Gulati, 1995; Eisenhardt \& Schoonhoven, 1996; Powell et al., 1996; Gulati, 1999;...). Néanmoins, peu d'études ont observé les alliances stratégiques à grande échelle et à partir d'une approche structurale (Gulati, 1998; Lazega, 2007; Borgatti et al., 2009). L'approche structurale est une méthode de modélisation du système d'interdépendance des acteurs dans un environnement, à travers un graphe. Une telle approche apporte un nouvel éclairage sur les modes d'organisation des alliances stratégiques.

Le but de cet article est donc d'étudier les marchés publics, pour identifier les réseaux d'alliances qui les composent. Nous tenterons, dans un premier temps, de présenter les fondements théoriques sur l'émergence des réseaux au sein des marchés. Ensuite, nous expliquerons la méthode utilisée pour capturer et étudier un grand nombre de relations d'alliances. Enfin, nous discuterons les résultats obtenus et proposerons une cartographie permettant de localiser des partenaires dans des réseaux stratégiques.

\section{Fondements théoriques}

\subsection{Stratégies d'accès aux marchés publics}

Les marchés publics sont des contrats ${ }^{2}$ conclus, à titre onéreux, entre un acheteur public (pouvoir adjudicateur) et un (ou des) opérateur(s) économique(s), pour répondre à ses besoins en matière : de travaux, de fournitures ou de services. Les marchés publics respectent les principes de liberté d'accès à la commande publique, d'égalité de traitement des candidats

\footnotetext{
${ }^{1}$ Marchés recensés en 2009 par l'Observatoire Économique de l'Achat Public

2 Pour plus d'informations sur le code des marchés publics, vous pouvez consulter le site Internet: http://www.economie.gouv.fr/directions_services/daj/marches_publics/oeap/index.htm
} 
et de transparence des procédures. Ces principes permettent d'assurer l'efficacité de la commande publique et la bonne utilisation des deniers publics.

Afin de garantir l'efficacité de la commande publique et la bonne utilisation des deniers publics, la passation des marchés publics doit comprendre : une définition préalable des besoins, un respect des obligations de mise en concurrence, et le choix de l'offre économiquement la plus avantageuse. Le libre accès à tous implique l'appel à la concurrence la plus large possible. L'équité de traitement repose sur des mécanismes d'attribution anonymes et non préférentiels, de façon à aboutir à des décisions impartiales et transparentes. En fait, l'attribution d'un appel d'offres correspond à une procédure d'enchère sous pli cacheté. La sélection de l'offre la plus intéressante se fait sur un ensemble de critères dont, notamment, le prix.

Pour comprendre le fonctionnement des marchés publics, Mougeot (1986) souligne que la passation des marchés de l'État et des collectivités locales est un problème relevant des relations « principal-agent» ou «mandant-mandataire ». En effet, dans ces procédures, l'acheteur public, en position de « principal », définit une règle d'attribution du marché face à laquelle les fournisseurs, en position d' " agents », déterminent leurs stratégies de soumission de propositions de prix, en situation d'incertitude sur les offres des concurrents. Lorsque plusieurs agents sont en relation avec un principal, l'incertitude de chacun sur le comportement des autres, s'ajoute aux asymétries d'information entre le principal et les agents. Chacun étant incité à utiliser ces asymétries pour atteindre son objectif au détriment des autres.

Alors, face à un appel d'offres, quels comportements stratégiques utilisent les soumissionnaires? Ils doivent réaliser un arbitrage entre deux tendances contradictoires : (1) soit ils offrent des prix élevés et diminuent leur chance de succès, mais ils obtiennent un profit élevé s'ils gagnent; (2) ils offrent des prix bas, et leurs chances d'emporter le marché sont élevées, mais leur profit est faible. Dans ce cas, ils doivent se prémunir contre la malédiction du vainqueur (Milgrom 1981), c'est-à-dire le fait de gagner, parce qu'ils ont mal estimé la vraie valeur de la demande.

Mais, dans tous les cas, et comme le précisent Mougeot et Naegelen (1990), la décision de participation à un appel d'offres résulte d'un calcul économique de comparaison du coût de réponse (certain) et du profit escompté (incertain). Ces auteurs montrent que dans un appel d'offres, le profit moyen du vainqueur dépend de la probabilité que l'un des concurrents propose une offre plus basse. Si tous les agents adoptent leur meilleure stratégie, ce profit décroît avec le nombre de concurrents. En conséquence, une orientation stratégique de soumission à un appel d'offres consiste à limiter l'intensité concurrentielle, en contrôlant les ressources nécessaires à la réalisation d'une transaction.

Cela nous conduit à formuler cette proposition de recherche : il existe des réseaux stratégiques, au sein des marchés publics, qui permettent à certaines entreprises de contrôler l'accès aux ressources.

\subsection{Apports de la sociologie économique}

Les relations interentreprises peuvent être considérées comme "un système coordonné d'acteurs hétérogènes, développant des transactions fondées sur des relations de coopération, afin de poursuivre collectivement un objectif partagé » (Voisin et al., 2004). Ce système est le résultat d'un processus au sein duquel les partenaires construisent des liens pour en tirer 
un bénéfice mutuel, c'est-à-dire un réseau composé d'acteurs, qui subit l'influence du milieu et l'oblige à trouver un équilibre avec son environnement. Comme le définit Assens (2003), le concept de réseau met donc l'accent sur l'interconnexion d'acteurs capables de participer à des échanges. Le lien entre les acteurs traduit la nature des échanges, leur périodicité, leur force, leur densité. Les acteurs occupent des positions qui sont susceptibles d'évoluer, mais qui témoignent d'un rôle ou d'une fonction assumées vis-à-vis des autres acteurs.

Pour comprendre la formation d'un réseau, de nombreux auteurs (Callon, 1989; Cohendet et Diani, 2003) privilégient l'étude des relations entre membres, plutôt que de se focaliser sur la nature des membres eux-mêmes. Ils critiquent, ainsi, une approche du réseau comme une forme d'organisation transitoire, au caractère hybride, entre le marché et la hiérarchie (Williamson, 1985).

C'est pourquoi, Granovetter (1985) considère que le fonctionnement d'un marché dépend des conditions sociales. Il partage ainsi, avec les autres auteurs de la sociologie économique (White, 1981 ; Baker, 1984), la conviction que l'action économique est une action sociale, orientée par des motivations diverses : la sociabilité, la reconnaissance, le statut social et le pouvoir. Pour rendre compte de l'insertion des actions économiques dans des systèmes de relations sociales, Granovetter (1985) propose le concept d' « encastrement structural ». Il s'efforce ainsi de démontrer que ce sont les relations sociales et les institutions qui permettent à un marché de fonctionner. Une relation est alors caractérisée par son contenu (l'échange d'une ressource), sa direction et sa force (intensité de l'échange).

Dans la même perspective, les relations interentreprises peuvent être considérées comme un moyen de contrôler l'incertitude de l'environnement dans l'accès à des ressources complémentaires. La performance d'une entreprise peut être comprise en examinant le réseau de relations dans lequel elle est encastrée. Ce « réseau stratégique » (Gulati et Nohria, 2000) correspond à l'ensemble des liens qu'elle peut entretenir avec ses partenaires, qu'ils soient clients, fournisseurs, concurrents ou sous-traitants.

D'ailleurs, la préférence à travailler avec un partenaire particulier dépend du niveau d'interactions suffisant pour arriver à une transaction satisfaisante, humainement, plutôt qu'économiquement, optimale (Voisin et al., 2004). Des acteurs reliés par des « liens forts » (Granovetter, 1983) supposent des contacts fréquents, une fourniture de services réciproques, et cela justifie le partage des ressources (Richardson, 1972). Selon cette approche, la relation sociale contribue à réguler les comportements opportunistes (Olson, 1971), à limiter les mesures de contrôles ou d'incitations (Gérard, 2000), à éviter l'asymétrie d'information entre partenaires (Akerlof, 1970), à réduire les coûts de transactions (Williamson, 1985) et à faciliter la résolution collective des problèmes. En effet, comme l'expliquent Gulati et Singh (1998), les acteurs économiques ont tendance à échanger, en priorité, avec des partenaires qu'ils connaissent, afin de réduire les incertitudes liées aux transactions.

Ainsi, les stratégies de coalitions, par l'échange d'informations qu'elles imposent et les réseaux qu'elles engendrent, peuvent faciliter la coordination, mais également favoriser la formation d'ententes horizontales entre les candidats potentiels à des appels d'offres publics. Ces ententes reposent souvent sur une répartition temporelle ou géographique des marchés, et peuvent prendre différentes formes. Comme l'explique Brisset (Brisset, 2003), ces concertations peuvent donner lieu au versement de paiements compensatoires, ou, de façon plus subtile et peut-être moins risquée, à des accords de sous-traitance, à des accords de répartition de lots, ou encore à une combinaison de prises de participation croisées et de rétrocessions monétaires entre sociétés membres de l'entente. 
Cela nous conduit à formuler cette nouvelle proposition de recherche : il existe des positions centrales au sein des réseaux stratégiques, qui permettent à certaines entreprises d'obtenir des avantages informationnels.

\section{Méthodologie}

\subsection{Analyse des réseaux}

L'analyse des réseaux sociaux (ou Social Network Analysis) considère la société comme un système d'acteurs sociaux reliés par des relations. L'un des principaux objectifs visés dans les études sur les réseaux, a été de comprendre la cohésion entre les membres comme un moyen d'accès, de partage ou de contrôle de ressources. Au-delà des explications opportunistes, liées notamment à la proximité (géographique ou sociale), deux grands principes permettent d'expliquer la formation des liens et les interactions (Lin et al., 2001). Lorsque des acteurs ont le même niveau de ressources et qu'ils sont motivés par le besoin de reconnaissance, d'échange ou de protection de leur statut social, on parle d'interactions homophiles. Dans ce cas, le but de la formation des liens est de maintenir son niveau de ressources. À l'inverse, lorsque les niveaux hiérarchiques sont différents et que les actions sont motivées par la nécessité d'obtenir des ressources meilleures ou supplémentaires, il s'agit d'interactions hétérophiles.

D'une façon plus générale, les acteurs interagissent avec d'autres acteurs, parce qu'ils recherchent des appuis ou des ancrages qui permettent de maîtriser leur environnement turbulent, et pour trouver une certaine stabilité. Cette recherche de contrôle engendre des régularités (relations) qui forment la base des réseaux sociaux. La structure de ces régularités peut être identifiée. C'est le principe de l'équivalence structurale (White, 1981). Comme le rappellent Grossetti et Godart (2007), deux entités sont structurellement équivalentes, au sein d'un réseau, si elles y occupent la même place, ou la même position, c'est-à-dire si elles ont les mêmes relations (ou des relations relativement similaires) avec un ensemble d'acteurs donné. La notion d'équivalence structurelle permet donc de retrouver la notion classique de rôle (ou de position), mais d'un point de vue strictement structurel, par une analyse des réseaux, sans faire d'hypothèse sur le contenu de ce rôle.

L'analyse des réseaux est donc une méthode adaptée pour comprendre et formaliser les phénomènes complexes faisant intervenir un système de relations interactif. En effet, cette méthode permet de décrire, et de reconstituer, un réseau, de façon simplifiée, à travers un graphe. Le graphe représente les interactions entre des objets reliés par des liens. Le développement d'une méthode quantitative, issue à l'origine de la sociométrie et complétée par les apports de la théorie des graphes, permet aujourd'hui de dégager un ensemble de propriétés qui forme une topologie modélisable (Guillaume et Latapy, 2003). Pour analyser les réseaux (Borgatti et al., 2009), trois grandes dimensions (Tableau 1) peuvent être retenues.

La première consiste à identifier les réseaux et à décrire la manière dont les structures de ces réseaux font peser des contraintes sur leurs membres. La connexité est un indicateur qui permet de définir la frontière du réseau au sein d'un graphe. Il y a un réseau, s'il existe toujours un chemin pour relier deux sommets de l'ensemble. Un réseau est donc une composante connexe d'un graphe.

La seconde dimension permet d'identifier la position, plus ou moins dominante, d'un acteur dans le réseau. Elle peut s'évaluer à partir des approches de centralité définies par 
Freeman (1979). La centralité de degré exprime la popularité d'un acteur dans le réseau, c'est-à-dire le nombre de connexions directes d'une entreprise aux autres. La centralité de proximité identifie les entreprises les plus proches des sources de pouvoir et d'influence, c'est-à-dire rapidement joignables par l'ensemble des autres membres du réseau.

La troisième dimension vise à dégager la cohésion des groupes homogènes au sein du réseau. Un groupe cohésif est un sous-ensemble d'acteurs entre lesquels on constate l'existence de relations fortes, intenses, directes et fréquentes. La mesure de la cohésion repose notamment sur l'analyse des similarités structurales dans le réseau. Deux acteurs sont donc structurellement équivalents s'ils ont des relations identiques avec les autres acteurs du réseau (White, 1981). Des techniques de partitionnement du réseau permettent de détecter des classes d'acteurs ayant une équivalence structurale (Navarro et Cazabet, 2011).

Tableau 1 : analyse des réseaux

\begin{tabular}{|l|l|l|}
\hline \multirow{3}{*}{ analyse des réseaux } & \multirow{2}{*}{ propriété des liens } & distance \\
\cline { 3 - 3 } & intensité relationnelle \\
\cline { 2 - 3 } & position des nouivalence structurale \\
\cline { 2 - 3 } & structure du réseau & centralité de degré \\
\cline { 2 - 3 } & centralité de proximité \\
\cline { 2 - 3 } & densité \\
\hline
\end{tabular}

\subsection{Terrain d'étude}

En novembre 2005, à l'initiative du ministre de l'économie, un observatoire de la commande publique (OEAP) a été mis en place. Son rôle est de publier et d'analyser les statistiques sur les marchés publics français. Sont ainsi recensés le nombre et le montant des marchés notifiés, par type et par année, depuis 2005. L'évaluation du marché des appels d'offres, notifiée en 2008, par type de marchés (travaux, services et fournitures) et par donneur d'ordre (État ou collectivités locales) peut être synthétisée dans le tableau 2 ci-dessous. Au final, la commande publique de l'État, des collectivités territoriales et des organismes publics, représentait plus de 68 milliards d'euros en 2008.

Tableau 2 : répartition des marchés publics français en 2008

\begin{tabular}{|l|r|r|r|}
\hline \multirow{2}{*}{ Donneur d'ordre } & Type de marché & $\begin{array}{c}\text { Nombre } \\
\text { de transactions }\end{array}$ & $\begin{array}{c}\text { Montant } \\
\text { des transactions }\end{array}$ \\
\hline \multirow{2}{*}{ État } & fournitures & 7506 & $17597406415 €$ \\
\cline { 2 - 4 } & travaux & 6271 & $5014175877 €$ \\
\cline { 2 - 4 } & services & 9469 & $13224158919 €$ \\
\hline Sous-total de l'État & & 23246 & $35835741211 €$ \\
\hline
\end{tabular}




\begin{tabular}{|l|r|r|r|}
\hline \multirow{2}{*}{ Collectivités locales } & fournitures & 30483 & $11610085661,00 €$ \\
\cline { 2 - 4 } & travaux & 41854 & $13864787682 €$ \\
\cline { 2 - 4 } & services & 18238 & $7216891262,00 €$ \\
\hline Sous-total des collectivités & 90575 & $32691764605,00 €$ \\
\hline Total général & $\mathbf{1 1 3 8 2 1}$ & $\mathbf{6 8 5 2 7 5 0 5 8 1 6 , 0 0} €$ \\
\hline
\end{tabular}

Les données utilisées pour cette étude concernent les réponses, par co-traitance, aux appels d'offres des marchés publics français. Elles sont issues du fichier des avis d'attribution du Bulletin Officiel des Annonces des Marchés Publics (BOAMP) des Journaux Officiels ${ }^{3}$. Le BOAMP publie les transactions attribuées par un donneur d'ordre public français, dont le montant du marché est supérieur à 4000 euros hors taxes.

À partir des transactions réalisées au cours de l'année 2008, nous avons sélectionné, avec des mots clés, les transactions réalisées uniquement dans le cadre de groupements d'entreprises. Le tableau 3 détaille le dénombrement de la population observée. Il précise le nombre d'acteurs que nous avons identifiés au sein des groupements.

Tableau 3 : caractéristiques de la population observée

\begin{tabular}{|l|c|}
\hline Nombre de transactions réalisées en totalité & 54181 \\
\hline Nombre de transactions réalisées par des groupements & 4203 \\
\hline Nombre d'entreprises dans les groupements & 6563 \\
\hline Nombre de liens de coopération & 10377 \\
\hline
\end{tabular}

L'obtention de l'ensemble de ces données est un processus de plusieurs étapes de traitement : extraction, nettoyage, filtrage, formatage, dédoublonnage et indexation.

La nature des données utilisées pour analyser les groupements d'entreprises, concerne trois variables. La première est un attribut lié à l'identification des acteurs (raison sociale). La deuxième concerne un attribut lié au type de bénéficiaires des transactions (groupements d'entreprises). La troisième variable concerne la relation de coopération qui relie les acteurs au sein d'un groupement. Nous avons considéré qu'il y a une relation de coopération entre deux entreprises lorsqu'elles obtiennent un marché dans le cadre d'une co-traitance (co-appartenance à un groupement d'entreprises).

\footnotetext{
${ }^{3}$ Les avis d'attribution du BOAMP sont accessibles en ligne $: \underline{w w w . j o u r n a l-o f f i c i e l . g o u v . f r}$
} 


\section{Résultats}

Nous avons organisé les données relationnelles sous la forme d'une liste d'adjacence. La liste énumère, pour chaque entreprise, toutes les entreprises qui lui sont voisines. Ensuite, nous avons utilisé la suite logicielle Graph $\mathrm{Viz}^{4}$, pour obtenir un graphe complet des relations de coopération au sein du marché public français en 2008.

\subsection{Détection des réseaux stratégiques}

Le graphe fait apparaître de nombreux sous-graphes (des composantes connexes) dans lesquels il existe un chemin entre tout couple de nœuds. Ces sous-graphes sont des réseaux stratégiques. Le graphe est composé de 1360 réseaux stratégiques ayant entre 2 et 2233 nœuds. La figure 1 présente la répartition des réseaux stratégiques en fonction de leur taille. La visualisation du graphe complet fait apparaître des agrégats d'entreprises qui correspondent à une concentration de liens sur un nombre réduit d'entreprises.

Figure 1 : distribution des réseaux en fonction de leur taille

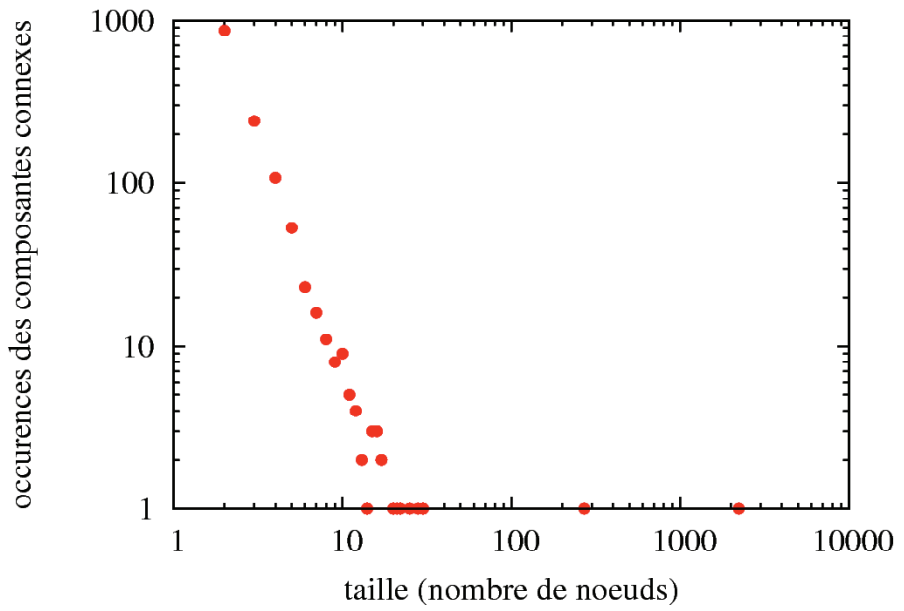

Pour comprendre la façon dont les réseaux stratégiques sont constitués, nous les avons isolés. L'étude approfondie du plus grand réseau stratégique, permet de produire un ensemble d'indicateurs sur la structure des relations :

- la densité globale du graphe est faible $(0,0004)$. Elle correspond au rapport entre le nombre de liens existant et le nombre de liens possibles;

- la densité locale, ou coefficient de clustering, est forte $(0,47)$. Elle correspond à la probabilité que deux voisins d'un même acteur soient reliés entre eux ;

- la distance moyenne entre deux entreprises est de 6,45. Cette distance correspond à la longueur des chaînes de relations entre des membres quelconques du réseau;

\footnotetext{
${ }^{4}$ Graphviz (www.graphviz.org) est un ensemble d'outils open source créé par les laboratoires de recherche d'AT\&T qui permet la représentation et l'analyse de graphes.
} 
- la distribution de la centralité de degré, c'est-à-dire le nombre de connexions qu'une entreprise a dans le réseau est hétérogène. La majorité des entreprises ont un degré faible, et quelques entreprises seulement ont un degré fort. Pour schématiser, nous sommes proches d'une loi de Zipf-Paretto où $20 \%$ des entreprises attirent et génèrent $80 \%$ des liens du réseau. On repère ces entreprises statistiquement, mais aussi visuellement dans le graphe.

\subsection{Organisation des réseaux stratégiques}

L'analyse du plus grand réseau stratégique dans les marchés publics français révèle des caractéristiques non-triviales communes dans d'autres réseaux sociaux, comme : les réseaux de connaissance (deux individus sont reliés s'ils se connaissent), les réseaux de contact physique (deux individus sont reliés s'ils ont été physiquement en contact), les réseaux de collaboration (deux individus sont reliés s'ils ont travaillé ensemble), les réseaux d'échanges (deux entités sont reliées si elles ont échangé un courrier électronique par exemple). En effet, tous les grands réseaux sociaux possèdent un ensemble de propriétés mathématiques qui ont été relevées lors de l'étude des grands graphes de terrain, avec une densité globale faible, une densité locale forte, des chemins courts entre les sommets, une hétérogénéité des degrés et un faible degré moyen. Ces caractéristiques sont généralement attribuées aux grands graphes de terrain (Strogatz, 2001), en référence à l'effet du «petit monde » de l'expérience de Travers et Milgram (1969). Ces caractéristiques constituent un modèle d'organisation des réseaux stratégiques.

La distribution des degrés dans le réseau montre l'existence de zones plus densément connectées que d'autres. Ces zones correspondent à des groupes d'entreprises plus fortement connectés entre eux qu'avec les autres. Elles correspondent à des ensembles d'entreprises possédant des points communs et dont les liens sont naturellement plus forts. La distribution de la fréquence des liens montre que le réseau est principalement organisé à partir de liens faibles. Or, comme l'a montré Granovetter (1983) pour les individus, ce sont les liens faibles qui permettent à l'information de circuler dans un réseau plus vaste. La force des liens faibles procure des informations qui ne sont pas disponibles dans un réseau restreint.

\section{Discussion}

\subsection{Cartographie des réseaux stratégiques}

D'un point de vue plus général, le graphe complet de la co-appartenance d'entreprises à des groupements d'entreprises nous offre un ensemble d'indicateurs pour décrire le phénomène de réseaux stratégiques au sein des marchés publics. Mais, pour exploiter ces indicateurs, nous avons besoin de déterminer, en permanence, la position qu'occupe chaque entreprise au sein du réseau, de comprendre son rôle et son importance dans le réseau, ou quelles sont les affinités permettant à une entreprise d'acquérir ou de conserver une position centrale dans le réseau.

Cette finalité rend indispensable l'utilisation d'une cartographie. La cartographie est composée des graphes égocentrés de chaque membre, avec ses caractéristiques topologiques. La carte représente l'espace stratégique où s'exercent les jeux d'influence, et la topologie permet de classer les entreprises en fonction de leurs propriétés relationnelles. La 
cartographie constitue, alors, un champ d'action réticulaire qui représente les interactions sociales des entreprises dans les marchés publics. Ce champ d'action est un espace dans lequel les acteurs communiquent des informations et interagissent les uns avec les autres.

Pour illustrer la cartographie que nous obtenons, la figure 2 représente un graphe égocentré de l'entreprise identifiée $N^{\circ} 1518$ avec son voisinage direct de 9 partenaires cotraitants. Elle a été extraite du réseau inter-organisationnel des marchés publics et permet d'identifier la position de l'entreprise par rapport à son environnement proche. Elle met en évidence les cliques et les clans auxquels participent l'entreprise. La carte montre l'espace stratégique dans lequel l'entreprise va pouvoir déployer son champ d'action réticulaire. Il permet de savoir, très simplement, qui travaille avec qui au sein du marché, et d'évaluer instantanément le niveau d'influence de chaque acteur.

Figure 2 : graphe égocentré de l'acteur 1518 du réseau

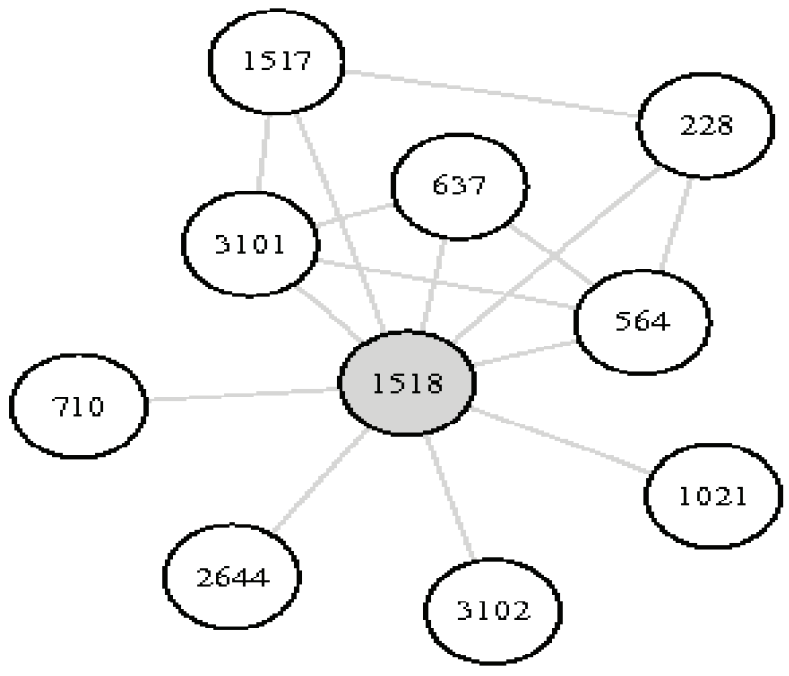

Au final, l'atout principal de la cartographie repose sur sa capacité à analyser, en permanence, les transactions et les relations au sein des marchés publics. Cette analyse des réseaux permet :

- de représenter les entreprises et leurs relations;

- de naviguer dans les réseaux d'entreprises;

- d'identifier la position de chaque entreprise dans le marché;

- de mesurer la force des liens (affinités) entre les entreprises;

- de déterminer le rôle et le statut de chaque entreprise.

\subsection{Vers un système d'intelligence économique}

La formation d'une alliance stratégique suppose de la part du dirigeant d'entreprise, une aptitude permanente à réagir à l'environnement, à s'adapter aux évolutions et à anticiper les changements. Face à l'environnement dynamique et instable, l'apport de l'intelligence 
économique peut être considéré comme la solution qui permettra la réduction de la part d'incertitude dans la prise de toute décision stratégique (Moinet, 2011). Comme le précise Morin (cité par Massé et al., 2006) : être intelligent, c'est pouvoir trouver une solution dans un environnement complexe. Cela correspond à la capacité d'absorption (Zahra et George, 2002) de l'information à des fins stratégiques, c'est-à-dire à son acquisition, son assimilation, sa transformation et son exploitation.

En effet, les entreprises qui voudront former des alliances stratégiques devront développer leur capacité à créer, et/ou activer, des relations de coopération dans le cadre d'un projet défini. Ces combinaisons de liens ne constituent qu'une matière première relationnelle. L'intelligence économique naît à partir du moment où l'un des acteurs, au moins, oriente et active ces liens dans le cadre d'un projet, et est à la recherche d'un avantage compétitif (Massé et al., 2006). D'ailleurs, El Haddadi (2012) précise que l'intelligence économique est à la fois un processus, un produit et un service. En tant que processus, l'intelligence économique permet le management stratégique de l'information. En tant que produit, il est un système organisationnel d'information stratégique. En tant que service, il offre une cartographie de l'environnement de l'entreprise.

L'intérêt d'une cartographie serait, alors, de fournir aux entreprises un système de recommandation de nouveaux liens, pour les aider à former des coalitions au sein des marchés publics. Mais pour concevoir un tel système, la démarche serait la suivante. À partir d'une étude longitudinale sur plusieurs années, nous pourrons prédire les nouveaux liens qui vont apparaître et qui relieront des entreprises, déjà présentes dans les réseaux, mais qui n'ont jamais été liées directement auparavant. Des techniques d'apprentissages supervisées (Benchettara, Kanawati et Rouveirol 2010) pourraient, alors, être appliquées pour construire un modèle de prédiction de nouveaux liens dans les réseaux, afin d'aider les entreprises à former des coalitions gagnantes.

Prendre la décision de former une coalition d'entreprises, revient à construire une structure particulière de liens au sein d'un système complexe de relations. La formation d'une coalition peut être considérée comme un jeu de décisions stratégiques, dans lequel les joueurs (les entreprises) peuvent se grouper pour obtenir des gains (transactions) en fonction de leurs choix (alliances temporaires) et en tenant compte de règles (implicites ou explicites) qui encadrent ou contraignent leurs comportements. Les gains dépendent de la décision d'acteurs qui ne font pas partie du jeu, et dont la distribution ne répond pas à une loi probabiliste connue (environnement incertain). Cette décision se définira par la prise en considération de multiples critères liés aux scores des propriétés relationnelles. Elle impliquera, entre autres, la diversité des options considérées, et l'évaluation en profondeur de chacune d'entre elles, en considérant les possibilités de gains.

\section{Conclusion}

Cette étude, réalisée à partir de données issues du BOAMP, est une analyse des marchés publics français durant l'année 2008. Ainsi, à partir de 54181 transactions entre les donneurs d'ordres et les entreprises soumissionnaires, nous avons pu observer 4203 transactions réalisées par des coalitions d'entreprises. L'enrichissement de ces transactions, avec des informations relationnelles, nous a permis d'effectuer une analyse structurale. De ce fait, nous constituons une base de données sur les relations interentreprises. 
La principale contribution de cette étude est de proposer un cadre d'analyse des réseaux stratégiques au sein des marchés publics. Nous réalisons une cartographie qui donne une représentation graphique des réseaux d'alliances. Elle facilite leur visualisation et révèle des caractéristiques non-triviales communes à d'autres réseaux sociaux.

Au-delà de la cartographie qui donne une représentation graphique des réseaux d'alliances, la finalité de cette étude est de proposer les bases d'un système d'intelligence économique. En effet, l'intelligence économique peut être considérée comme un processus qui a pour objet la réduction de la part d'incertitude dans la prise de toute décision stratégique (Moinet, 2011).

Néanmoins, nous devons tenir compte du fait que la coalition est un lieu où se nouent et se dénouent des interactions sociales. Elles sont susceptibles d'évoluer, mais également de disparaître. Aussi, il nous faut continuer cette étude. Pour ce faire, nous avons comme ambition de suivre, de façon dynamique, les évolutions et mouvements des réseaux d'alliances dans les marchés publics, sur une période de plusieurs années. L'étude longitudinale tentera de comprendre l'émergence et l'évolution des réseaux stratégiques au sein des marchés publics.

\section{Bibliographie}

Akerlof G., 1970. The Market for "Lemons", Quality Uncertainty and the Market Mechanism. Quarterly Journal of Economics 84, 353-374.

Assens C., 2003. Le réseau d'entreprises, vers une synthèse des connaissances. Management international 7 (4), 49-59.

Baker W.E., 1984. The Social Structure of National Securities Market. American Journal of Sociology 96 (3), 589-625.

Benchettara N., Rushed K. et Rouveirol C., 2010. Apprentissage supervisé pour la prédiction de nouveaux liens dans des réseaux sociaux bipartie. In Actes da la $17^{\mathrm{e}}$ Rencontre de la société francophone de classification SFC2010, 63-66.

Borgatti S., Ajay M., Brass, D. et Labianca G., 2009. Network Analysis in The Social Sciences. Science 323 (5916), 892-5.

Brisset, K., 2003. Ententes horizontales dans les procédures d'enchères et d'appels d'offres. Revue française d'économie 18 (2), 111-164.

Callon M. 1989. La science et ses réseaux, genèse et circulation des faits scientifiques. la Découverte, Paris.

Charreaux G. 2000. La théorie positive de l'agence, positionnement et apports. Revue d'Économie Industrielle 92, 193-214.

Cohendet P. et Diani M., 2003. L'organisation comme une communauté de communautés croyances collectives et culture d'entreprise. Revue d'économie politique 113, 697-720.

Eisenhardt K., et Schoonhoven C., 1996. Resource-based View of Strategic Alliance Formation, Strategic and Social Effects in Entrepreneurial Firms. Organization Science 7 (2), 136-150.

El Haddadi A., 2012. Le système d'intelligence économique. VSST. Ajaccio, Actes du colloque.

Freeman L.C., 1985. Centrality in Social Networks Conceptual Clarification. Social Networks 1, 215-239.

Granovetter M., 1985. Economic Action and Social Structure, the Problem of Embeddedness. American Journal of Sociology 91 (3), 481-510.

Granovetter M., 1983. The Strength of Weak Ties, a Network Theory Revisited. Sociological Theory 1, 201-233.

Grossetti M. et Godart F., 2007. Harrison White, des réseaux sociaux à une théorie structurale de l'action. SociologieS. 
Guillaume J.-L. et Latapy M., 2003. Modèles pour les topologies réalistes. Proceedings of ALGOTEL'03 (Cinquièmes Rencontres Francophones sur les aspects Algorithmiques des Télécommunications).

Gulati R., 1999. Network Location and Learning, The Influence of Network Resources and Firm Capabilities on Alliance Formation. Strategic Management Journal 20 (5), 397-420.

Gulati R., 1995. Does Familiarity Breed Trust? The Implications of Repeated Ties for Contractual Choice in Alliance. Academy of Management Journal 38 (1), 85-112.

Gulati R. et Nohria N., 2000. Strategic Networks. Strategic Management Journal 1-34.

Gulati R. et Singh H., 1998. The Architecture of Cooperation : Managing Coordination Costs and Appropriation Concerns in Strategic Alliances. Adminitrative Science Quartely 43 (4), 781-814.

Jolly D., 2001. Alliances interentretrepises, entre concurrence et coopération, Vuibert, Paris.

Kogut B., 1988. Joint Ventures, Theoretical and Empirical Perspectives. Strategic Management Journal 9, 319-332.

Lazega E., 2007. Réseaux sociaux et structures relationnelles, PUF, Paris.

Lemieux V., 1998. Les Coalitions, Liens, transactions et contrôles. PUF, Paris.

Lin N., Cook K. and Burt R. 2006. Social Capital, Theory and Research. AldineTransaction.

Massé G., Marcon C. et Moinet N., 2006. Les fondements de l'intelligence économique, réseaux et jeu d'influence. Market Management.

Milgrom P. , 1981. Good News and Bad News, Representation Theorem and Applications. Bell Journal of Economics 12, 380-391.

Moinet N., 2011. Intelligence économique, mythes et réalités. CNRS, Paris.

Mougeot M., 1986. Marchés publics, règles rigides ou procédures flexibles? Politiques et management public $4(3), 1-25$.

Mougeot M. et Naegelen F. 1990. La libéralisation des marchés publics. Revue d'Économie Industrielle 52, 52-68.

Navarro E. et Cazabet R., 2011. Détection de communautés, étude comparative sur graphes réels. In Collectif, Information interaction intelligence Vol. 11 (1), 77-93.

Olson M., 1971. Logic of Collective Action, Public Goods and the Theory of Groups. Harvard University Press, Cambridge.

Powell W., Koput K., and Smith-Doerr L., 1996. Interorganizational Collaboration and the Locus of Innovation, Networks of Learning In Biotechnology. Administrative Science Quarterly 41(1), 116-145.

Richardson G. 1972. The Organization of Industry. Economic Journal 82, 883-896.

Strogatz S H., 2001. Exploring Complex Networks. Nature 410 (6825), 268-76.

Travers J. and Milgram S. 1969. An Experimental Study of the Small World Problem. Sociometry 32 (4), $425-443$.

Voisin C., Ben Mahmoud-Jouini S. et Édouard S., 2004. Les réseaux, dimensions organisationnelles et stratégiques. Economica, Paris.

White H., 1981. Where do Markets Come From? American Journal of Sociology 87 (3), 517-58.

Williamson O., 1985. The Economic Institutions of Capitalism. Free Press-Collier Macmillan. Basingstoke.

Zahra S. A. and George G. , 2002. Absorptive Capacity, a review, Reconceptualization, and Extension. Academy of Management Review 27 (2), 185-203. 
